\title{
Special Issue: Capsule Networks and Imaging Science (CNIS)
}

\author{
P. Karuppusmay ${ }^{1} \cdot$ Fuqian $\mathrm{Shi}^{2} \cdot$ Yong Shi $^{3}$
}

Published online: 3 June 2021

(c) The Author(s), under exclusive licence to Springer Science+Business Media, LLC, part of Springer Nature 2021

Imaging science has emerged as one of the most significant research domains by developing new technologies for image generation, analysis, classification, and visualization. The current technologies like big data, artificial intelligence [AI], big data, machine learning and deep learning enables a detailed real-time visualization of the intended images. To give a newer dimension to the imaging science, the term capsule networks have been conceptualized. With its advanced capability to fetch the spatial information and more significant image features, Capsule Networks (CapsNet) can easily overcome any information loss that happen during the image processing tasks especially during image reconstruction. This special issue on "Capsule Networks and Imaging Science" has investigated the challenges in real-time image processing and proposed novel solutions with different neural network models to process the complex data and tasks.

We are pleased to present this special issue on Capsule Networks and Imaging Science. It contains some of the latest research works that streamlines the research into problems at the intersection of neural networks and imaging science.

This special issue contains 15 state-of-the-art research articles, where first article proposes an extreme learning machine (ELM) method for establishing advanced fault diagnosis in fuel systems along with a Bat algorithm. The proposed research work has been validated by considering their improved classification accuracy and generalization ability. The second paper discusses about the Semi-supervised learning approach for machine learning to combine the labeled and unlabeled data, in particular this paper proposes a safe semisupervised classification algorithm using multiple classifiers ensemble (S3C-MC). When the whole experiment is carried out on the UCI dataset, the result shows that S3C-MC has good safety and higher classification rate. The third paper focuses on the biomedical imaging science particularly the lung cancer prediction using a novel feature selection algorithm that is wrapper-based and proposed by employing the modified stochastic diffusion search

P. Karuppusmay

pkarrupusamy@ieee.org

Fuqian Shi

fs446@cinj.rutgers.edu

Yong Shi

yshi5@kennesaw.edu

1 Department of EEE, Shree Venkateshwara Hi-Tech Engineering College, Gobichettipalayam, India

2 Rutgers Cancer Institute of New Jersey, New Brunswick, USA

3 Kennesaw State University, Kennesaw, USA 
(SDS) algorithm. The results of the proposed research work deliver better performance when compared to existing methods. The fourth paper takes the initiative to respond to the rapid change of the demand brought by the urban change. A virtual system of 3D historical block based on VR is designed, and the public facilities are designed in the virtual system. Moreover, BP neural network is designed to simplify the process of design evaluation and program modification and greatly improve the design efficiency of public facilities in historical blocks. The fifth paper proposes an efficient mammogram image retrieval system called Modified Adaptive Neuro-Fuzzy Inference System optimized by ABC Algorithm ('Artificial Bee Colony') to establish advanced breast cancer prevention into existence. The proposed model is evaluated by utilizing disparate database images. Furthermore, in order to provide intelligent solution to analyze the effects of traditional public security management and various new types of criminal activities, the sixth paper proposes an intelligent crime prevention and control model based on big data analysis, machine Internet of Things and industrial object system in order to deliver high data collection rate and crime prevention and control efficiency. The seventh paper deeply investigates the multimedia technologies to perform complex data management based on multimedia database technology. In this research perspective, this paper proposes a self-organizing capsule neural network and game theory to deliver higher efficiency and robustness in the performing the image processing tasks. With the rapid development of image processing and computer vision technology, it paves way to process the collected video image automatically without human intervention. To achieve this, the eighth paper proposes a city monitoring system based on real-time communication interaction module and intelligent visual information collection system to incorporate strong timeliness and good monitoring effect. The primary research objective of the ninth paper is to develop a novel methodology for brain tumor segmentation. To achieve this, a combination of Artificial Neural Network [ANN] and Fuzzy K-means algorithm has been presented to segment the tumor locale. Wherein, the overall accuracy of this proposed technique has been improved by $8 \%$ when compared to other existing models. The tenth paper proposes a canonical correlation analysis based hyper basis feed forward neural network classification (CCA-HBFNNC) model for evaluating sustainable urban environmental quality to select the key pollutant features, which bear fundamental implications to the modernize air pollution. In addition, the proposed model also minimizes the time complexity of urban development when contrasted with contemporary works. Furthermore, the automatic segmentation of the tumor region from Magnetic Resonance cerebrum imageries is a difficult task in medical image analysis, to overcome this challenge, the eleventh paper proposes a 2D-ConvNet with skull stripping (SS-2D ConvNet) based brain tumor segmentation technique to reduce noise and skull stripped in order to correct the contrast and non-uniformity and achieve better performance and yield precise segmentation. The twelfth paper aims to maintain the product quality, production operation in a continuous and stable state and real-time monitoring of product quality in the industrial production process by proposing a visual imaging technology and text analysis to build an efficient soft computing model based on Bayesian network. The thirteenth paper introduces three-dimensional network reconstruction method and three-dimensional vision technology, and puts forward the visualization scheme of urban landscape ecological design to meet the normal traffic function of the road and realize the beautiful green landscape. The fourteenth paper proves that deep learning can learn more complex knowledge, when compared with the traditional image recognition methods. As a real-time application, this paper proposes a medical burn image recognition system by using convolutional neural network technology and deep learning. With the experimental results, it is proved that the proposed model delivers a better robustness when compared with existing algorithms. 
Lastly, the fifteenth paper provides the real-time application of Meta-learning Framework based on multi-intelligent nervous system for performing image classification and recognition. Also, this research work integrates the multiple-capsule intelligent neural systems to construct the efficient model in order to obtain higher image recognition rate.

We hope the readers will relish this extensive research analysis on imaging science, machine learning, deep learning and neural networks.

Publisher's Note Springer Nature remains neutral with regard to jurisdictional claims in published maps and institutional affiliations. 